\title{
Fault Tolerant Localization for Teams of Distributed Robots
}

\author{
Renato Tinós \\ Department of Electrical Engineering \\ EESC \\ University of Sao Paulo \\ São Carlos, SP 13560-970, Brazil \\ tinos@sel.eesc.sc.usp.br
}

\begin{abstract}
To combine sensor information from distributed robot teams, it is critical to know the locations of all the robots relative to each other. This paper presents a novel fault tolerant localization algorithm developed for centimeterscale robots, called Millibots. To determine their locations, the Millibots measure the distances between themselves with an ultrasonic distance sensor. They then combine these distance measurements with dead reckoning in a maximum likelihood estimator.

The focus of this paper is on detecting and isolating measurement faults that commonly occur in this localization system. Such failures include dead reckoning errors when the robots collide with undetected obstacles, and distance measurement errors due to destructive interference between direct and multi-path ultrasound wave fronts.

Simulations show that the fault tolerance algorithm accurately detects erroneous measurements and significantly improves the reliability and accuracy of the localization system.
\end{abstract}

Keywords: mobile robotics; fault tolerance; collaborative localization; robot teams; fault detection and isolation.

\section{Introduction and Related Work}

No longer confined to industrial applications, robots are more often entering the human environment. Toy robots, robotic wheelchairs, surgical robots, and robots in hospitals and nursing homes all come in close contact with humans. Some research suggests that, by 2010, the number of robots in homes will reach 5 million [5]. As a result, a failure in a robotic system can not only cause unacceptable economic losses but also put the safety of the people in its environment at risk [16].

This situation is aggravated by the fact that robot failures are relatively common. Even in well-structured

\author{
Luis E. Navarro-Serment and Christiaan J.J. Paredis \\ Institute for Complex Engineered Systems \\ Dept. of Electrical and Computer Engineering \\ Carnegie Mellon University \\ Pittsburgh, Pennsylvania 15213-3890 \\ \{lenscmu, paredis\}@cmu.edu
}

industrial environments, the recorded mean time to failure for manipulators ranges from only 500 to 2500 hours [4]. We expect that robot failures will be even more common in household robots for which, most likely, rigorous preventive maintenance schedules will not be enforced. According to Parker [13], it is due to the lack of research on fault tolerance and adaptivity of robot teams that robot autonomy and multi-robot cooperation have not yet been adequately demonstrated.

This paper investigates fault tolerance issues in a group of robot systems that are particularly vulnerable to failures. The CMU Millibots are very small $(7 \times 7 \times 7 \mathrm{~cm})$ and inexpensive robots that contain little or no redundancy within each robot but rely on collaboration within a team to identify and overcome failures. These Millibots provide a good test-bed for fault tolerance as they reflect the limited reliability and capabilities of future inexpensive household robots.

Fault tolerance is usually achieved in two steps: The system first detects and isolates the faults, after which it reconfigures itself to overcome the faults. Generally, for individual mobile robots, this approach requires considerable redundancy in sensing, actuation, communication, and computation, resulting in large, complex, and expensive systems. For multi-robot systems, redundancy is also available at the team level. For instance, sensing capabilities on one robot may be replaced by a combination of sensing modalities on other robots. As a result, fault tolerance can still be implemented for robots with limited capabilities (and possibly without any redundancy) such as the Millibots.

In this paper, the problem of fault tolerance in the localization system of the CMU Millibots is addressed. The objective of the Millibots is to collaboratively map and explore an unknown environment. When a robot moves, its new position is estimated from a combination of dead reckoning measurements and distance measurements between robots. Faults in these measurements produce incorrect position estimates, and 
correspondingly, errors in the maps of the environment. This paper presents a method to detect and overcome such errors, based on the information redundancy in the dead reckoning and distance measurements.

\section{The Millibots}

The Millibots are configured from modular components including communication, computation, mobility, camera, sonar, and IR modules [8]. Assembling different types of modules creates specialized robots that collaborate to accomplish a given task. Because of their small size $(7 \times 7 \times 7 \mathrm{~cm})$, the computational and sensing capabilities of Millibots are limited. Higher-level functions such as mapping and localization are provided by a larger robot or team leader.

The knowledge of the position and orientation of each Millibot is crucial to achieve accurate mapping and exploration of the environment. Conventional localization systems, based on dead reckoning, GPS, landmark recognition or map-based positioning [15], do not offer a viable solution due to limitations in size, power, or sensing of the Millibots. To overcome these problems, a novel method was developed that utilizes dead reckoning and ultrasonic distance measurements between robots [12].

The Millibot localization system is based on trilateration [2], i.e., determination of the position based on distance measurements to known landmarks or beacons $[9,10]$. GPS is an example of a trilateration system; the position of a GPS unit on earth is calculated from distance measurements to satellites in space. Similarly, the Millibot localization system determines the position of each robot based on distance measurements to stationary robots with known positions.

As is illustrated in Figure 1, the distance between two

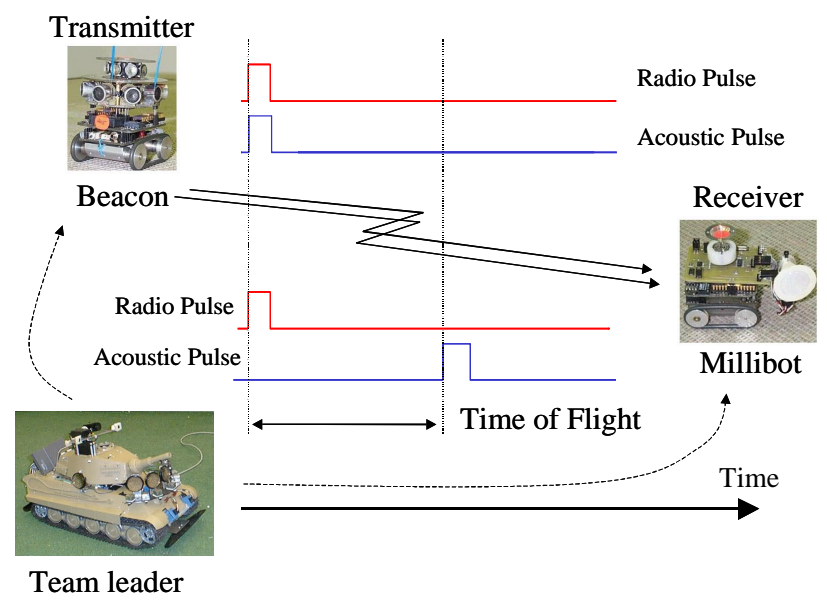

Figure 1: Ultrasonic distance measurement. robots is measured using synchronized ultrasound and RF pulses. A conical reflector mounted above a low-cost transducer allows the Millibots to detect and transmit ultrasonic pulses in any direction [8]. Periodically, each robot that serves as a beacon emits simultaneously a radio frequency (RF) pulse and an ultrasonic pulse. Using the RF pulse for synchronization, the distance to the beacon is measured as the time-of-flight of the ultrasonic pulse multiplied by the speed of sound $\left(343 \mathrm{~m} / \mathrm{s}\right.$ at $\left.20^{\circ} \mathrm{C}\right)$. The team leader coordinates the pinging sequence to ensure that beacon signals from multiple robots do not interfere with one another. To improve the accuracy, this procedure is repeated several times and the sample mean is utilized to estimate the distance to the beacon.

All the Millibots transmit their distance measurements to the team leader who calculates the new robot positions. A maximum likelihood algorithm determines the most likely position of the robot given the measured distances to the current beacons. Assuming that the dead reckoning and distance measurements are normally distributed random variables, the likelihood of being located at a position $(x, y)$ is given by

$$
\begin{aligned}
& P\left(x, y \mid x_{d}, y_{d}, \bar{r}_{b}(1), \bar{r}_{b}(2), \cdots, \bar{r}_{b}(m)\right)= \\
& \quad N\left(x-x_{d}, \sigma_{x}^{2}\right) N\left(y-y_{d}, \sigma_{y}^{2}\right) \prod_{i=1}^{m} N\left(r(i)-\bar{r}_{b}(i), \sigma_{b}^{2}\right)
\end{aligned}
$$

where $N\left(p, \sigma^{2}\right)$ is a normal distribution with zero mean and variance of $\sigma^{2}$ evaluated at $p,\left(x_{d}, y_{d}\right)$ is the position measured through dead reckoning, $r(i)$ is the distance from the beacon $i$ to the Millibot, $m$ is the number of beacons, and $\bar{r}_{b}(i)$ is the sample mean of the distance measurements from beacon $i$ to the Millibot.

The estimated new position of the Millibot is given by the value of $(x, y)$ that maximizes the probability density function in Equation (1), and is computed using the BFGS non-linear optimization algorithm [6]. The algorithm is initialized with the dead reckoning estimate $\left(x_{d}, y_{d}\right)$ or an estimate based on the closed form trilateration expression derived in [11]. In general, only a few iterations are necessary to reach the optimum value because of its proximity to the starting point.

\section{Fault Modes and Effects Analysis}

Although the localization algorithm described above has the potential to provide very accurate position estimates [12], practice has shown that it is susceptible to multiple failures, some of which occur relatively often. Before developing a fault tolerance scheme, we analyze these failure modes using Fault Modes and Effects Analysis (FMEA) [1]. 


\subsection{Incorrect ultrasonic distance measurements}

We have identified three different causes for erroneous distance measurements.

A first failure occurs when the ultrasonic pulse is either not emitted by the beacon or not received by the Millibot. This could happen due to a failure in the transducers, the circuitry, or the communication with the team leader. These faults do not occur often and result in a clearly identifiable effect: failing to register an ultrasonic pulse.

Incorrect distance measurements also result when there is an obstacle between the beacon and the Millibot blocking the ultrasonic pulse. The effect, in this case, is more complicated because an ultrasonic pulse that bounces around the obstacle (multi-path) may still be detected, resulting in a distance measurement larger than the actual distance.

The same effect can occur due to destructive interference. As is illustrated in Figure 2, at certain distances between the robots, the wave propagated in the direct path interferes destructively with the wave that bounces off the floor. For the Millibots this failure mode is especially pronounced at distances of $0.5 \mathrm{~m}$ and $0.8 \mathrm{~m}$ (the interference at $2.5 \mathrm{~m}$, shown in Figure 2, is outside the range of the sensor). The effect, again, is that a secondary echo is measured instead, resulting in too large a distance measurement.

\subsection{Incorrect dead reckoning measurements}

The motion commands for the Millibots are executed by a local PID controller that receives feedback from optical encoders. Although the control loop is sufficiently accurate to ensure that the motors actually execute the desired movement (within a certain variance due to thread slippage), the following failures can still occur.

A first group of faults is a result of hardware failures of the actuators, mechanical transmissions, wheels, encoders or controllers. These faults are rather uncommon and
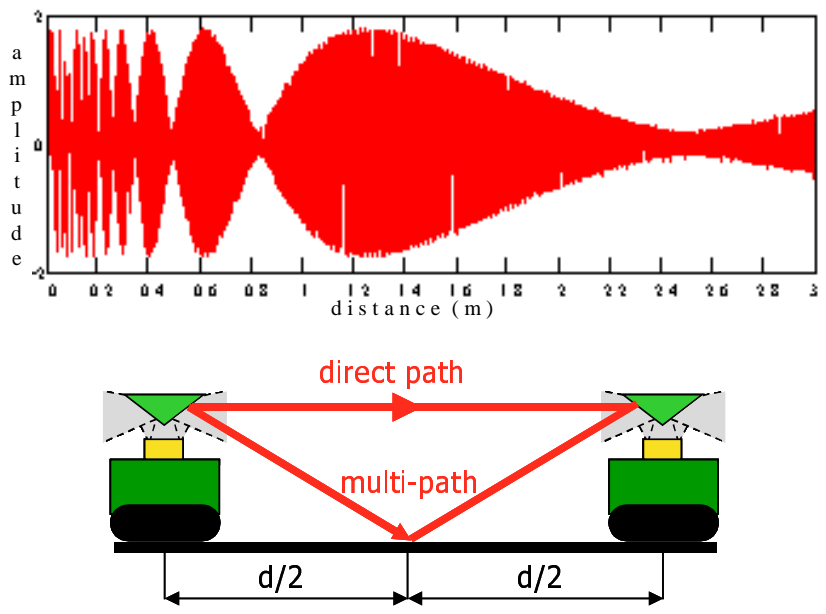

Figure 2: Destructive interference. result in a movement to the wrong position, no movement at all, or continuous movement without stopping. A more common failure mode occurs when the Millibots run into an undetected obstacle. The effect varies from stopping before reaching the desired position to falling over. Due to thread slippage, these failures cannot be detected through the encoder readings.

In conclusion, both groups of faults result in a discrepancy between the actual position and the estimated position based on dead reckoning.

\section{Fault Tolerance}

As pointed out in the previous section, faults affecting localization occur commonly in the Millibot systemespecially the destructive interference failure mode occurs often. To ensure accurate position estimation, it is critical that these faults are detected and isolated so that they can be taken into account by the estimation algorithm. Such a fault tolerance scheme is presented in this section.

\subsection{Fault Detection and Isolation}

Past research in fault detection and isolation (FDI) has focused on faults in individual mobile robots with redundant sensors $[7,14]$. For example, encoder readings are compared with integrated gyroscope measurements to detect faulty estimates of the robot orientation. The Millibots, however, do not have this level of sensing redundancy. Instead, they take advantage of the information redundancy in the combined dead reckoning and ultrasonic distance measurements for the entire team of robots.

Based on the dead reckoning information, we can compute the expected distance from the moving Millibot to each of the beacon Millibots. Assuming that the distance traveled and the distance to the beacons is relatively large, this expected distance is approximately normally distributed. The ultrasonic distance measurement is also a sample from a population that is approximately normally distributed (the discretization error in the ultrasonic sensor is much smaller than the measurement error). Our FDI scheme is based on statistical tests that verify whether the two normally distributed distance measurements (based on dead reckoning and ultrasonic pulses) are consistent, i.e., have the same expected value. If they are not consistent, a fault has occurred.

The test is based on the following statistical properties. Consider two random variables, $\left(x_{1}, x_{2}\right)$, from two different populations both with a normal distribution. It can be shown that the difference of the sample means, $\left(\bar{x}_{1}-\bar{x}_{2}\right)$, is also normally distributed with a mean and variance equal to [3]: 


$$
\mu_{\bar{x}_{1}-\bar{x}_{2}}=\mu_{1}-\mu_{2} \quad \sigma_{\bar{x}_{1}-\bar{x}_{2}}=\sqrt{\frac{\sigma_{1}^{2}}{n_{1}}+\frac{\sigma_{2}^{2}}{n_{2}}}
$$

where $n_{1}$ and $n_{2}$ are the sample sizes. Or, as a direct corollary:

$$
z=\frac{\left(\bar{x}_{1}-\bar{x}_{2}\right)-\mu_{\bar{x}_{1}-\bar{x}_{2}}}{\sigma_{\bar{x}_{1}-\bar{x}_{2}}}=\frac{\left(\bar{x}_{1}-\bar{x}_{2}\right)-\left(\mu_{1}-\mu_{2}\right)}{\sqrt{\sigma_{1}^{2} / n_{1}+\sigma_{2}^{2} / n_{2}}}
$$

is normally distributed with zero mean and unit variance.

To test whether both populations, $x_{1}$ and $x_{2}$, have the same expected value $\left(\mu_{1}=\mu_{2}\right)$, we can use the following hypothesis:

$$
H_{0}: \mu_{1}-\mu_{2}=0 \text { if }-z_{1-\alpha / 2} \leq z \leq z_{1-\alpha / 2}
$$

where $\alpha$ is the level of significance, and $z$ is

$$
z=\frac{\left(\bar{x}_{1}-\bar{x}_{2}\right)}{\sqrt{\sigma_{1}^{2} / n_{1}+\sigma_{2}^{2} / n_{2}}}
$$

An alternative hypothesis is

$$
H_{1}: \mu_{1}-\mu_{2} \neq 0 \text { if } z \leq-z_{1-\alpha / 2} \text { or } z \geq z_{1-\alpha / 2} .
$$

For a given $\alpha$, the value of $z_{1-\alpha / 2}$ can be found in the standard normal tables.

We now apply this test to the two distance measurements: $r_{d}(i)$, the distance from the beacon $i$ to the Millibot based on dead reckoning, and $r_{b}(i)$, the corresponding ultrasonic distance measurement. The hypothesis variable, $z(i)$, is then:

$$
z(i)=\frac{r_{d}(i)-\bar{r}_{b}(i)}{\sqrt{\sigma_{r_{d}(i)}^{2}+\sigma_{b}^{2} / n_{b}(i)}} \quad, \quad i=1,2, \cdots, m
$$

where $n_{b}(i)$ is the number of independent ultrasonic measurements by beacon $i$. Assuming that the variances on the position coordinates, $x$ and $y$, are small, the variance $\sigma_{r_{d}(i)}^{2}$ can be obtained from:

$$
\sigma_{r_{d}(i)}^{2}=\left(\frac{\partial r_{d}(i)}{\partial x}\right)^{2} \sigma_{x}^{2}+\left(\frac{\partial r_{d}(i)}{\partial y}\right)^{2} \sigma_{y}^{2} .
$$

Table 1 summarizes the different fault scenarios and corresponding statistical hypotheses: If no faults occur, the variable $z(i)$ of every beacon is small, confirming the hypothesis $H_{0}$. If an error in the ultrasonic distance measurement between the Millibot and beacon $j$ occurs (hypothesis $\left.H_{I}\right), z(j)$ is negative and large (negative

\begin{tabular}{|c|c|c|}
\hline Hyp. & Fault & Region \\
\hline$H_{0}$ & No faults & $\begin{array}{c}-z_{1-\alpha / 2} \leq z(i) \leq z_{1-\alpha / 2} \\
\text { for } \mathrm{i}=1,2, \ldots, \mathrm{m}\end{array}$ \\
\hline$H_{1}$ & $\begin{array}{c}\text { Incorrect } \\
\text { ultrasonic distance } \\
\text { measurement for } \\
\text { beacon } \mathrm{j}\end{array}$ & $\begin{array}{c}-z_{1-\alpha / 2} \geq z(j) \\
-z_{1-\alpha / 2} \leq z(i) \leq z_{1-\alpha / 2} \\
\text { for } \mathrm{i}=1,2, \ldots, \mathrm{m}, \mathrm{i} \neq \mathrm{j}\end{array}$ \\
\hline$H_{2}$ & $\begin{array}{c}\text { Incorrect dead } \\
\text { reckoning } \\
\text { information }\end{array}$ & otherwise \\
\hline
\end{tabular}

Table 1: Hypothesis tests for the FDI procedure.

because an erroneous measurement is always larger than the true distance, as explained in Section 3). Since we assume that only one error occurs at a time, the other variables, $z(i)$ with $i \neq j$, will all be small. If this is not the case, then we conclude that an error in the dead reckoning measurement has occurred (hypothesis $\mathrm{H}_{2}$ ).

\subsection{Reconfiguration}

After the fault has been detected and isolated, the localization algorithm is easily reconfigured by ignoring the erroneous measurement. If an incorrect distance measurement for beacon $j$ is detected, Equation (1) is modified to

$$
\begin{aligned}
& P\left(x, y \mid x_{d}, y_{d}, \bar{r}_{b}(1), \bar{r}_{b}(2), \cdots, \bar{r}_{b}(m)\right)= \\
& \quad N\left(x-x_{d}, \sigma_{x}^{2}\right) N\left(y-y_{d}, \sigma_{y}^{2}\right) \prod_{\substack{i=1 \\
i \neq j}}^{m} N\left(r(i)-\bar{r}_{b}(i), \sigma_{b}^{2}\right)
\end{aligned}
$$

If incorrect dead reckoning measurements are detected, Equation (1) becomes

$$
P\left(x, y \mid \bar{r}_{b}(1), \bar{r}_{b}(2), \cdots, \bar{r}_{b}(m)\right)=\prod_{i=1}^{m} N\left(r(i)-\bar{r}_{b}(i), \sigma_{b}^{2}\right)
$$

in which the dead reckoning information is not utilized. This requires that at least three Millibots serve as beacons for a unique maximum to exist.

\section{Results}

A series of experiments have been conducted to test the effectiveness of a team of Millibots to explore and map a given area [8]. In these experiments, a human operator who controlled the Millibot team could plan the individual robot motions to avoid distances at which destructive interference occurs. The robot motions were also planned to avoid ill-conditioned configurations, such as collinear beacons. The experiments showed that the localization algorithm performs well when no faulty distance measurements occur. However, it becomes very difficult 


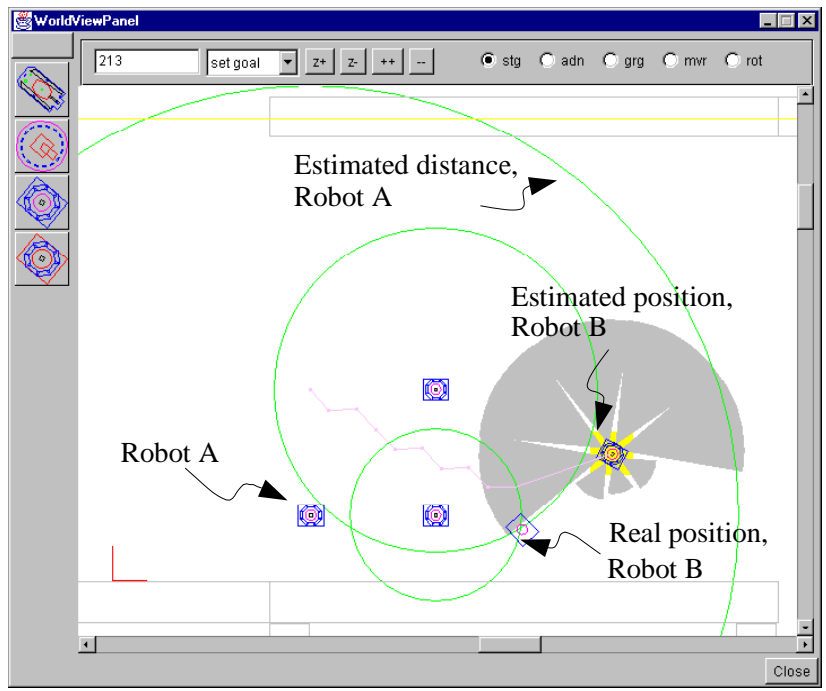

Figure 3: Faulty position estimation for Robot $B$ when an erroneous ultrasonic measurement occurred with respect to beacon $A$.

to avoid these faults (i.e. avoid collinearity and destructive interference) with more than four robots on a team.

We performed several simulations to test the fault tolerance algorithms. The Figures 3 and 4 show snapshots from a GUI that controls four Millibots in a mapping task. The simulations performed without the fault tolerance system (Figure 3) show that significant errors result when incorrect distance estimates are considered in the localization algorithm. The gray region around the robot $B$ indicates the area covered by the sonar sensors used to detect objects; errors in the estimated position translate into significant mapping errors.

The simulations in which the fault tolerance system was

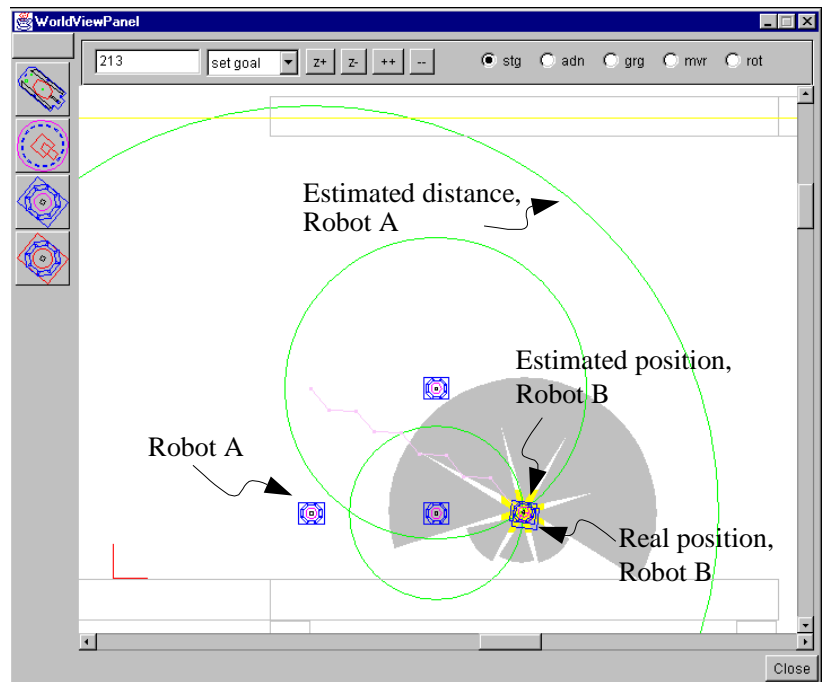

Figure 4: Correct position estimate for robot $B$. The FDI procedure detected the erroneous distance measurement with respect to beacon $A$. active show that the faults described in Section 3 could be detected correctly. One such scenario is illustrated in Figure 4. In this simulation, destructive interference occurred in the distance measurement between the robots $\mathrm{A}$ and B. The values of $z$ in Equation (7) were -6.03 for beacon $\mathrm{A}$, and 0.41 and -0.04 for the other beacons. For a significance of $\alpha=0.01, z_{1-\alpha / 2}$ equals 2.326 resulting in a confirmation of hypothesis $H_{l}$ (since $-6.03<-2.326$ ). As a result, the localization algorithm ignored the erroneous distance measurement to robot $\mathrm{A}$ and estimated the robot position based only on the dead reckoning information and the distance measurements to the other two robots.

The fault tolerance algorithm has the additional advantages that it is not computationally expensive, and that it provides additional quantitative information to the human operator with respect to the performance of the localization system. This information can be used to improve the individual robot motion operation.

\section{Conclusion}

Due to destructive interference of ultrasonic pulses or collisions with undetected obstacles, faults occur commonly in the Millibot localization system. It is therefore important to employ fault tolerance mechanisms to improve its reliability and accuracy.

This paper introduced an FDI system based on statistical hypothesis testing that can identify which of the measurements (distance measurements and dead reckoning) is incorrect. Because of the structure of the maximum likelihood estimator, the localization algorithm can be easily modified to omit these erroneous measurements. Simulations showed that the fault tolerance procedure successfully detected and compensated for incorrect measurements, thus improving the accuracy and reliability of the localization system.

In the future, the fault tolerance procedure should be tested with the real Millibots. In particular, research should focus on incorporating of fault tolerance procedures into the real-time Millibot path-planning system. The problem of multiple simultaneous faults should be addressed too, along with a characterization of the reliability of the localization system under this scenario.

\section{Acknowledgements}

The authors would like to thank Robert Grabowski for his contribution. The Millibots project is funded in part by the Distributed Robotics program of DARPA/ETO under contract DABT63-97-1-0003 and, by the Institute of Complex Engineered Systems at Carnegie Mellon 
University. Renato Tinós is supported by FAPESP under grant 98/15732-5.

\section{References}

[1] Birolini, A., Reliability Engineering: Theory and Practice, Third ed. New York: Springer, 1999.

[2] Borenstein, J., Everett, H. R., and Feng, L., Navigating Mobile Robots: Sensors and Techniques. Wellesley, MA: A.K. Peters, 1996.

[3] Chase, W. and Bown, F., General Statistics, Fourth ed. New York: John Wiley \& Sons, 2000.

[4] Dhillon, B. S., Robot Reliability and Safety. New York: Springer-Verlag, 1991.

[5] Dhillon, B. S. and Fashandi, A. R. M., "Robotic Systems Probabilistic Analysis," Microelectronics and Reliability, vol. 37, pp. 211-224, 1997.

[6] Fletcher, R., Practical Methods of Optimization, Second ed. New York: John Wiley \& Sons, 1987.

[7] Goel, P., Dedeoglu, G., Roumeliotis, S. I., and Sukhatme, G. S., "Fault Detection and Identification in a Mobile Robot Using Multiple Model Estimation and Neural Network," IEEE International Conference on Robotics and Automation, Leuven, Belgium, 1998.

[8] Grabowski, R., Navarro-Serment, L. E., Paredis, C. J. J., and Khosla, P. K., "Heterogeneous Teams of Modular Robots for Mapping and Exploration," Autonomous Robots, vol. 8, pp. 293-308, 2000.

[9] Kleeman, L., "Optimal Estimation of Position and Heading for Mobile Robots Using Ultrasonic Beacons and Dead-Reckoning," 1992 IEEE
International Conference on Robotics and Automation, Nice, France, pp. 2582-2587, 1992.

[10] Leonard, J. F. and Durrant-Whyte, H. F., "Mobile Robot Localization by Tracking Geometric Beacons," IEEE Transactions on Robotics and Automation, vol. 7, pp. 376-382, 1991.

[11] Manolakis, D. E., "Efficient Solution and Performance Analysis of 3-D Position Estimation by Trilateration," IEEE Transactions on Aerospace and Electronic Systems, vol. 32, pp. 1239-1248, 1996.

[12] Navarro-Serment, L. E., Paredis, C. J. J., and Khosla, P. K., "A Beacon System for the Localization of Distributed Robotic Teams," International Conference on Field and Service Robotics, Pittsburgh, pp. 232-237, 1999.

[13] Parker, L. E., "ALLIANCE: An Architecture for Fault Tolerant Multi-Robot Cooperation," IEEE Transactions on Robotics and Automation, vol. 14, pp. 220-240, 1998.

[14] Roumeliotis, S. I., Sukhatme, G. S., and Bekey, G. A., "Sensor Fault Detection and Identification in a Mobile Robot," 1998 IEEE/RSJ International Conference on Intelligent Robots and Systems, Victoria, Canada, pp. 1383-1387, 1998.

[15] Stuck, E. R., Manz, A., Green, D. A., and Elgazzar, S., "Map Updating and Path Planning for Real-Time Mobile Robot Navigation," 1994 IEEE/RSJ International Conference on Intelligent Robots and Systems, Munich, Germany, pp. 753-360, 1994.

[16] Visinsky, M. L., Cavallaro, J. R., and Walker, I. D., "Robotic Fault Detection and Fault Tolerance: A Survey," Reliability Engineering and System Safety, vol. 46, pp. 139-158, 1994. 